\title{
MÉTODOS PARA ESTIMATIVA DA EVAPOTRANSPIRAÇÃO DE REFERÊNCIA PARA CHAPADÃO DO SUL - MS
}

Fernando França da Cunha ${ }^{1}$, Fernando Fagner Magalhães ${ }^{2}$, Marco Aurélio de Castro²

\section{RESUMO}

A evapotranspiração é um dos parâmetros de maior incerteza no gerenciamento de recursos hídricos e manejo da irrigação. Antes da escolha de determinado método para estimativa da evapotranspiração de referência (ETo) em uma região, é de extrema importância avaliar o grau de exatidão do modelo. A presente pesquisa objetivou avaliar o desempenho de 30 métodos para estimativa da ETo para Chapadão do Sul, MS. Os dados meteorológicos foram retirados do Instituto Nacional de Meteorologia no período de quatro anos (2008 a 2011). O método tomado como padrão foi o Penman-Monteith-FAO56; e a comparação dos resultados foi por meio do coeficiente de determinação $\left(r^{2}\right)$, dos coeficientes "a" e "b" das regressões lineares, erro-padrão da estimativa (EPE), índice de concordância de Willmott (d), coeficiente de correlação de Pearson (r) e do coeficiente de confiança (c). Os melhores métodos para estimativa da ETo foram: Penman-Original, Stephens-Stewart, Radiação-Temperatura, Turc, Hargreaves-Original, Penman-FAO24, Thornthwaite, Abtew, Blaney-Criddle-FAO24, McCloud, Radiação-FAO24, Garcia-Lopez, Thornthwaite-Modificado, Radiação-Global, Hargreaves-Samani e Jensen-Haise. Quando se tem apenas dados de temperatura, recomenda-se utilizar o método de McCloud. O método de Hargreaves-Samani poderá ser utilizado depois de sua equação receber calibração para a região de estudo. Os métodos Makkink, Priestley-Taylor, Camargo, Linacre, Hamon, Ivanov, Kharrufa, Blaney-Morin, McGuiness-Bordne, Romanenko, Lungeon, Hicks-Hess, RadiaçãoLíquida e Tanner-Pelton não devem ser utilizados para a estimativa da ETo em Chapadão do Sul.

Palavras-chave: agrometeorologia, ETo, evaporação, irrigação, Penman-Monteith-FAO56.

\begin{abstract}
METHODS FOR ESTIMATING REFERENCE EVAPOTRANSPIRATION FOR CHAPADÃO DO SUL - MS

One of the parameters of greatest uncertainty related to water and irrigation management is evapotranspiration. Before selecting the determined method to estimate the reference evapotranspiraton (ETo) in a region, it is extremely important to evaluate the degree of precision of the model. The objective of the present study was to evaluate the performance of 30 methods to estimate the ETo for Chapadão do Sul, Brazil. Meteorological data was obtained from the National Institute of Meteorology during a four year period (2008 to 2011). The standard method was Penman-Monteith-FAO56 and comparison of the results was performed by means of the determination coefficient $\left(r^{2}\right)$, the coefficients " $a$ " and " $b$ " of the linear regressions, the estimated standard error (ESE), the Willmott concordance index (d), Pearson correlation coefficient (r) and confidence coefficient (c). The best methods for estimating ETo were obtained by the Penman, Stephens-Stewart, Radiation-Temperature, Turc, Hargreaves, Penman-FAO24, Thornthwaite, Abtew, Blaney-Criddle-FAO24, McCloud, Radiation, Garcia-Lopez, Modify-Thornthwaite, Global-Radiation, Hargreaves-Samani and Jensen-Haise methods. The McCloud method is recommended when only temperature data is used. The Hargreaves-Samani method can be used after calibrating its equation for the region of study. The Makkink, Priestley-Taylor, Camargo, Linacre, Hamon, Ivanov, Kharrufa, Blaney-Morin, McGuiness-Bordne, Romanenko, Lungeon, Hicks-Hess, Liquid-Radiation and Tanner-Pelton methods cannot be applied to estimate the ETo in Chapadão do Sul, Brazil.
\end{abstract}

Keywords: agrometeorology, ETo, evaporation, irrigation, Penman-Monteith-FAO56.

Recebido para publicação em 20/01/2012. Aprovado em 06/03/2013.

1 - Agrônomo, Doutor em Engenharia Agrícola, Professor Adjunto, UFMS/Chapadão do Sul, MS. E-mail: fernando.cunha@ufms.br

2 - Graduando Agronomia, UFMS/Chapadão do Sul, MS. E-mail: magalhaes.fernandof@gmail.com

3 - Graduando Agronomia, UFMS/Chapadão do Sul, MS. E-mail: falecomarco@yahoo.com 


\section{INTRODUÇÃO}

A estimativa adequada da evapotranspiração da cultura (ETc) consiste no principal parâmetro a ser considerado no dimensionamento e manejo de sistemas de irrigação, uma vez que totaliza a quantidade de água utilizada nos processos de evaporação e transpiração pela cultura durante determinado período (OLIVEIRA et al., 2001).

A ETc é obtida por meio de coeficientes relacionados à planta, ao solo, às condições atmosféricos e da evapotranspiração de referência (ETo). O método de Penman-Monteith (ALLEN et al., 1998) foi recomendado pela FAO como o padrão para calcular a ETo e tem sido utilizado em todo o mundo. Este método exige vários parâmetros de entrada como temperatura do ar, umidade relativa, radiação solar e velocidade do vento. Entretanto, há um número limitado de estações meteorológicas para monitoramento dessas variáveis do tempo. Esta falta de dados meteorológicos leva ao desenvolvimento de abordagens mais simples para estimar a ETo que exigem apenas alguns parâmetros de entrada. Neste contexto, vários métodos têm sido relatados na literatura para estimar a ETo.

Apesar da existência de diversos modelos de estimativa da ETo, no entanto, são utilizados em condições climáticas e agronômicas muito diferentes daquelas em que inicialmente foram concebidos e, por isso, é de extrema importância avaliar o grau de exatidão desses modelos, antes de utilizá-los para nova condição. Diversos trabalhos comparando os vários métodos de estimativa da ETo são encontrados na literatura, para diferentes regiões (BRAGANÇA et al., 2010; MENDONÇA; DANTAS, 2010; TAGLIAFERRE et al., 2010; ALENCAR et al., 2011; CAVALCANTE Jr. et al., 2011).

A região de Chapadão do Sul tem grande importância econômica devido à agricultura, tendo o segundo maior Produto Interno Bruto do Estado de Mato Grosso do Sul (SOBEL et al., 2009). O Município é um dos maiores produtores de soja, algodão e milho do País e possui duas usinas de açúcar e álcool com grande área cultiva de cana de açúcar (CHAPADÃO DO SUL, 2013). Por isso, a determinação correta da ETo é de extrema importância, visando o planejamento de recursos hídricos e ao manejo da agricultura irrigada da região.

Diante do exposto, objetivou-se com este trabalho, avaliar o desempenho de 30 métodos para estimativa da ETo, apontando os melhores e piores métodos em relação ao de Penman-MonteithFAO56, para o Município de Chapadão do Sul, MS.

Quadro 1. Metodologias e suas respectivas equações para estimativa da evapotranspiração de referência (ETo) utilizada na pesquisa

\begin{tabular}{|c|c|}
\hline Metodologia & Equação \\
\hline Penman-Monteith-FAO56 (ALLEN et al., 1998) & $E T_{0}=\frac{0,408 s\left(R_{N}-G\right)+\gamma \frac{900}{t+273} U_{2} \frac{\left(e_{S}-e\right)}{10}}{s+\gamma\left(1+0,34 U_{2}\right)}$ \\
\hline Penman-Original (PENMAN, 1948) & $E T_{0}=\frac{s}{s+\gamma} 0,408\left(R_{N}-G\right)+\frac{\gamma}{s+\gamma} 0,26\left(1+\frac{U_{2}}{160}\right)\left(e_{S}-e\right)$ \\
\hline Penman-FAO24 (DOORENBOS e PRUITT, 1977) & $E T_{0}=c\left[\frac{s}{s+\gamma} 0,408 R_{N}+\frac{\gamma}{s+\gamma} 0,27\left(1+0,864 U_{2}\right)\left(e_{S}-e\right)\right]$ \\
\hline Blaney-Criddle-FAO24 (DOORENBOS e PRUITT, & $E T_{0}=k p(0,457 t+8,13)$ \\
\hline Radiação-FAO24 (DOORENBOS e PRUITT, 1977) & $E T_{0}=-0,3+k\left(\frac{s}{s+\gamma} R_{G \mathrm{~mm} / \mathrm{dia}}\right)$ \\
\hline Makkink (MAKKINK, 1957) & $E T_{0}=R_{G \text { mm/dia }}\left(\frac{s}{s+\gamma}\right)+0,12$ \\
\hline Hargreaves-Samani (HARGREAVES e SAMANI, 19 & $E T_{0}=0,0023 R_{0 \text { mm/dia }}\left(t_{\max }-t_{\min }\right)^{0,5}(t+17,8)$ \\
\hline Hargreaves-Original (HARGREAVES, 1974) & $E T_{0}=0,135 \frac{R_{G}}{\lambda}(t+17,8)$ \\
\hline
\end{tabular}


Priestley-Taylor (PRIESTLEY e TAYLOR, 1972)

$$
E T_{0}=0,5143 \frac{s}{s+\gamma}\left(R_{N}-G\right)
$$

Jensen-Haise (JENSEN e HAISE, 1963)

$$
E T_{0}=R_{G \text { mm/dia }}(0,025 t+0,08)
$$

Camargo (CAMARGO, 1971)

$$
E T_{0}=0,01 R_{0 \mathrm{~mm} / \text { dia }} t
$$

Linacre (LINACRE, 1977)

$$
E T_{0}=\frac{500(t+0,006 z)}{100-\phi}+15\left(t-t_{d}\right) /(80-t)
$$

Hamon (HAMON, 1961)

$$
E T_{0}=0,55\left(\frac{N}{12}\right)^{2}\left(\frac{4,95 \exp ^{0,062 t}}{100}\right) 25,4
$$

Ivanov (DORFMAN, 1977)

$$
E T_{0}=0,006(25+t)^{2}\left(1-\frac{f}{100}\right)
$$

Kharrufa (KHARRUFA, 1985)

$$
E T_{0}=0,34 p t^{1,3}
$$

Garcia-Lopez (GARCIA e LOPEZ, 1970)

Blaney-Morin (BLANEY e MORIN, 1942)

$$
\begin{array}{r}
E T_{0}=1,2110^{\left(\frac{7,45 t}{243,7+t}\right)}(1-0,01 f)+0,21 t-2,30 \\
E T_{0}=p(0,457 t+8,13)(1,14-0,01 f)
\end{array}
$$

Turc (TURC, 1961) $E T_{0}=\frac{0,013 t}{t+15}\left(23,9 R_{G}+50\right)$

McCloud (JACOBS e SATTI, 2001) $E T_{0}=0,254 \quad 1,07^{(1,8 t)}$

McGuiness-Bordne (OUDIN et al., 2005) $E T_{0}=\frac{R_{0}}{\lambda} \frac{t+5}{68}$

Romanenko (OUDIN et al., 2005) $E T_{0}=4,5\left(1+\frac{t}{25}\right)^{2}\left(1-\frac{e}{e_{S}}\right)$

Lungeon (ZIMMERMANN, 2000) $E T_{0}=0,2985\left(e_{S}-e\right)\left(\frac{273+t}{273}\right)\left(\frac{760}{P-e_{S}}\right)$

Abtew (OUDIN et al., 2005) $E T_{0}=\frac{0,53}{\lambda} R_{G}(1-r)$

Hicks-Hess (BRUIN e KEIJMAN, 1979)

Radiação-Global (TOMAR e O'TOOLE, 1980)

Radiação-Líquida (BRUIN e STRICKER, 2000)

Radiação-Temperatura (OUDIN et al., 2005)

Stephens-Stewart (WINTER et al., 1995) $E T_{0}=0,4047 R_{G}[(0,01476 t)+0,0724]$

Tanner-Pelton (BERLATO e MOLION, 1981) $E T_{0}=0,457 R_{N}-0,11$

Thornthwaite-Modificado (CAMARGO et al., 1999)

$$
\begin{array}{r}
E T_{0}=\frac{16}{30}\left(10 \frac{0,36\left(3 t_{\max }-t_{\min }\right)}{I}\right)^{a} \frac{N}{12} \\
E T_{0}=\frac{16}{30}\left(10 \frac{t_{i}}{I}\right)^{a} \frac{N}{12}
\end{array}
$$

Thornthwaite (THORNTHWAITE, 1948)

ETo $=$ evapotranspiração de referência $\left(\mathrm{mm} \mathrm{dia}^{-1}\right) ; \mathrm{s}=$ declividade da curva de pressão $\left(\mathrm{kPa}^{\circ} \mathrm{C}^{-1}\right) ; \mathrm{R}_{\mathrm{N}}=$ saldo de radiação $\left(\mathrm{MJ} \mathrm{m}^{-2}\right.$ dia $\left.^{-1}\right) ; \mathrm{G}=$ fluxo de calor $\left(\mathrm{MJ} \mathrm{m}^{-2} \mathrm{dia}^{-1}\right) ; \gamma=$ constante psicrométrica $\left(\mathrm{kPa}^{\circ} \mathrm{C}^{-1}\right) ; \mathrm{t}=$ temperatura média $\left({ }^{\circ} \mathrm{C}\right) ; \mathrm{U}_{2}=$ velocidade do vento $\left(\mathrm{m} \mathrm{s}^{-1}\right) ; \mathrm{e}_{\mathrm{S}}=$ pressão de saturação de vapor $(\mathrm{hPa})$; e = pressão de vapor $(\mathrm{hPa}) ; \mathrm{c}=$ coeficiente de ajuste $(\mathrm{adm}) ; \mathrm{k}=$ coeficiente local $(\mathrm{adm}) ; \mathrm{p}=$ percentagem anual de luz (\%); $\mathrm{f}=$ umidade relativa (\%); $\mathrm{R}_{\mathrm{G} \text { mm/dia }}=$ radiação global $\left(\mathrm{mm} \mathrm{dia}^{-1}\right) ; \mathrm{R}_{0 \mathrm{~mm} / \text { dia }}=$ radiação extraterrestre $\left(\mathrm{mm} \mathrm{dia}^{-1}\right) ; \mathrm{t}_{\max }=$ temperatura máxima $\left({ }^{\circ} \mathrm{C}\right) ; \mathrm{t}_{\min }=$ temperatura mínima $\left({ }^{\circ} \mathrm{C}\right) ; \lambda=$ calor latente de vaporização $\left(\mathrm{MJ} \mathrm{kg}^{-1}\right) ; \mathrm{z}=$ altitude do local $(\mathrm{m}) ;=$ latitude do local (graus); $\mathrm{t}_{\mathrm{d}}=$ temperatura do ponto de orvalho $\left({ }^{\circ} \mathrm{C}\right) ; \mathrm{N}=$ fotoperíodo $(\mathrm{h}) ; \mathrm{R}_{\mathrm{G}}=$ radiação global $\left(\mathrm{MJ} \mathrm{m}^{-2}\right.$ dia $\left.^{-1}\right) ; \mathrm{R}_{0}=$ radiação extraterrestre $\left(\mathrm{MJ} \mathrm{m}^{-2} \operatorname{dia}^{-1}\right)$; $\mathrm{P}=$ pressão atmosférica $(\mathrm{hPa}) ; \mathrm{a}=$ constante do local $(\mathrm{adm}) ; \mathrm{I}=$ índice térmico anual $(\mathrm{adm})$ e $\mathrm{t}_{\mathrm{i}}=$ temperatura mensal $\left({ }^{\circ} \mathrm{C}\right)$. 


\section{MATERIAL E MÉTODOS}

Os dados meteorológicos necessários para execução desse trabalho foram retirados do Instituto Nacional de Meteorologia (Inmet), para a estação meteorológica do Município de Chapadão do Sul, MS (Latitude $18^{\circ} 48^{\prime} 08^{\prime \prime} \mathrm{S}$, Longitude $52^{\circ} 36^{\prime} 10^{\prime \prime}$ $\mathrm{W}$, Altitude de $818 \mathrm{~m}$ ), no período de quatro anos, compreendido entre janeiro de 2008 a dezembro de 2011. O clima é classificado pelo método de Köppen como tropical úmido, a temperatura anual fica compreendida entre 13 a $29^{\circ} \mathrm{C}$, a precipitação pluviométrica média é de $1.850 \mathrm{~mm}$, com concentração de chuva no verão e seca no inverno.

Os dados horários meteorológicos utilizados na presente pesquisa foram: temperatura instantânea, máxima e mínima $\left({ }^{\circ} \mathrm{C}\right)$; umidade relativa instantânea, máxima e mínima (\%); temperatura do ponto de orvalho instantânea, máxima e mínima $\left({ }^{\circ} \mathrm{C}\right)$; pressão instantânea, máxima e mínima (hPa); velocidade do vento $\left(\mathrm{m} \mathrm{s}^{-1}\right)$ e radiação global $\left(\mathrm{kJ} \mathrm{m}^{-2}\right)$.

Os dados meteorológicos horários foram convertidos para dados diários. Em seguida, foi realizado o preenchimento de falhas por meio do método de ponderação regional com base em regressão linear. Com o propósito de tornar os dados das variáveis meteorológicas mais homogêneos, foi feita uma verificação e posteriormente eliminação daquelas informações consideradas discrepantes ou inconsistentes visando obter agrupamentos de dados mais representativos.

As metodologias utilizadas na presente pesquisa para estimativa da evapotranspiração de referência (ETo) estão apresentadas no Quadro 1.

Após a verificação dos dados foi realizada uma análise de regressão que correlacionou os valores de ETo estimados pelas equações empíricas com o método Penman-Monteith-FAO56 (ALLEN et al., 1998). Foram considerados os coeficientes "a" $e$ " $b$ " das respectivas regressões lineares simples e o coeficiente de determinação $\left(r^{2}\right)$. A melhor alternativa foi aquela que apresentou coeficiente de regressão "a" próximo de zero, coeficiente "b" próximo da unidade e maior coeficiente de determinação. A precisão é dada pelo coeficiente de determinação, pois indica o grau em que a regressão explica a soma do quadrado total.

A análise de desempenho dos modelos foi feita comparando os valores deETo obtidos pelos métodos empíricos com o método de Penman-MonteithFAO56 (ALLEN et al., 1998). A metodologia adotada para comparação dos resultados foi proposta por Allen et al. (1989), e se fundamenta no erropadrão da estimativa (EPE), calculada pela Equação 1. O melhor método para estimativa da ETo foi aquele que apresentou o menor EPE.

$E P E=\left[\frac{\sum_{i=1}^{n}\left(Y_{i}-X_{i}\right)^{2}}{n}\right]^{1 / 2}$

em que

$\mathrm{EPE}=$ erro-padrão da estimativa $\left(\mathrm{mm} \mathrm{d}^{-1}\right)$;

$\mathrm{Y}_{\mathrm{i}}=$ evapotranspiração de referência estimada pelo método-padrão $\left(\mathrm{mm} \mathrm{d}^{-1}\right)$;

$\mathrm{X}_{\mathrm{i}}=$ evapotranspiração de referência obtida pelo método testado $\left(\mathrm{mm} \mathrm{d}^{-1}\right)$; e

$\mathrm{n}=$ número de observações.

A aproximação dos valores de ETo estimados por determinado método estudado, em relação aos valores obtidos com uso do método padrão, foi obtida por um índice designado concordância ou ajuste, representado pela letra "d" (WILLMOTT et al., 1985), onde seus valores variam desde zero, onde não existe concordância, a 1 , para a concordância perfeita. $\mathrm{O}$ índice de concordância (d) foi calculado aplicando-se a Equação 2. Para validação do modelo, obteve-se também o coeficiente de correlação de Pearson (r) pela Equação 3 e o coeficiente de confiança ou desempenho (c) pela Equação 4.

$$
\begin{aligned}
& d=1-\frac{\sum_{i=1}^{n}\left(Y_{i}-X_{i}\right)^{2}}{\sum_{i=1}^{n}\left[\left(\left|Y_{i}-\bar{X}\right|\right)+\left(\left|X_{i}-\bar{X}\right|\right)^{2}\right.} \\
& r=\frac{\sum_{i=1}^{n}\left(\mid X_{i}-\bar{X}\right)\left(\mid Y_{i}-\bar{Y}\right)}{\sqrt{\sum_{i=1}^{n}\left(X_{i}-\bar{X}\right)^{2}} \sqrt{\sum_{i=1}^{n}\left(Y_{i}-\bar{Y}\right)^{2}}} \\
& c=r d
\end{aligned}
$$

em que

d = índice de concordância ou ajuste de Willmott; $Y_{i}=$ evapotranspiração de referência estimada pelo método-padrão $\left(\mathrm{mm} \mathrm{d}^{-1}\right)$;

$\mathrm{X}_{\mathrm{i}}=$ evapotranspiração de referência obtida pelo

\section{REVENG}


método testado $\left(\mathrm{mm} \mathrm{d}^{-1}\right)$;

$\bar{X}=$ média dos valores de evapotranspiração de referência obtidos pelo método testado $\left(\mathrm{mm} \mathrm{d}^{-1}\right)$;

$\mathrm{n}=$ número de observações;

$\mathrm{r}=$ coeficiente de correlação de Pearson;

$\bar{Y}=$ média dos valores de evapotranspiração de referência obtidos pelo método-padrão $\left(\mathrm{mm} \mathrm{d}^{-1}\right)$ e; $\mathrm{c}=$ coeficiente de confiança ou desempenho.

Segundo Cohen (1988), o coeficiente de correlação (r) pode ser classificado como: "muito baixo" $(\mathrm{r}<0,1)$; "baixo" $(0,1<\mathrm{r}<0,3)$; "moderado" $(0,3<\mathrm{r}<0,5)$; "alto" $(0,5<\mathrm{r}<0,7)$; "muito alto" $(0,7<\mathrm{r}<0,9)$; e "quase perfeito" $(\mathrm{r}>0,9)$.

O coeficiente c, proposto por Camargo e Sentelhas (1997), é interpretado de acordo com os referidos autores como: "ótimo" (c > 0,85); "muito bom" $(0,76$ $<\mathrm{c}<0,85)$; "bom" $(0,66<\mathrm{c}<0,75)$; "mediano" $(0,61$ $<\mathrm{c}<0,65)$, "sofrível" $(0,51<\mathrm{c}<0,60)$, "mau" $(0,41$ $<\mathrm{c}<0,50)$ e "péssimo" $(\mathrm{c}<0,40)$.

\section{RESULTADOS E DISCUSSÃO}

Na Figura 1 encontram-se os valores diários de temperatura, umidade relativa, radiação global e velocidade do vento obtidos durante o período estudado. Os valores diários de radiação global variaram entre 8,0 e $31,4 \mathrm{MJ} \mathrm{m}^{-2} \mathrm{dia}^{-1}$, sendo seus valores mínimos ocorridos nos meses entre maio e agosto, e máximos nos meses compreendidos entre novembro e fevereiro. Esse comportamento influenciou os valores de temperatura e, consequentemente, os valores de umidade relativa do ar. Os valores médios de temperatura do ar durante o período estudado variaram de 12,9 a $29,2{ }^{\circ} \mathrm{C}$, sendo máximos entre os meses de outubro e março e mínimos entre os meses de abril e setembro. Os valores médios de umidade relativa variaram entre 20 e $93 \%$. O comportamento da umidade relativa foi o oposto da radiação solar e da temperatura, observando-se valores máximos entre os meses de outubro e março e mínimos entre os meses de abril e setembro. Os valores médios mensais de velocidade do vento variaram de 0,3 a $4,4 \mathrm{~m} \mathrm{~s}^{-1}$.

Nas Figuras 2 e 3 estão apresentados os gráficos e os modelos resultantes da regressão linear considerando os métodos de estimativa da evapotranspiração de referência (ETo) utilizados na análise tendo o método de Penman-Monteith padronizado pela FAO como padrão. Observase, com base na reta de regressão, que apenas o método de Blaney-Morin subestimou os valores de ETo, independente da estimativa de PenmanMonteith-FAO56, corroborando com Peña e Peña (2010) e Ejieji (2011). O método de BlaneyCriddle-FAO24 subestimou os valores de ETo apenas quando o método de Penman-MonteithFAO56 acusou estimativas acima de $4,5 \mathrm{~mm} \mathrm{mia}^{-}$ 1. Já os métodos de Priestley-Taylor, Camargo, Abtew e Radiação-Líquida subestimaram ETo quando os valores de Penman-Monteith-FAO56 acusaram estimativas superiores a $3,0 \mathrm{~mm} \mathrm{dia}^{-1}$, os métodos de Hamon, Hicks-Hess e Radiação-Global superiores a 2,5 $\mathrm{mm} \mathrm{dia}^{-1}$, e Stephens-Stewart e Thornthwaite-Modificado superiores a 2,0 $\mathrm{mm}$ dia- $^{-}$ ${ }^{1}$. Já os métodos Makkink, Jensen-Haise, Linacre, Kharrufa e McGuiness-Bordne se destacaram por apresentar altos coeficientes de regressão "a" e "b", ou seja, esses métodos superestimaram os valores de ETo em relação ao método padrão.

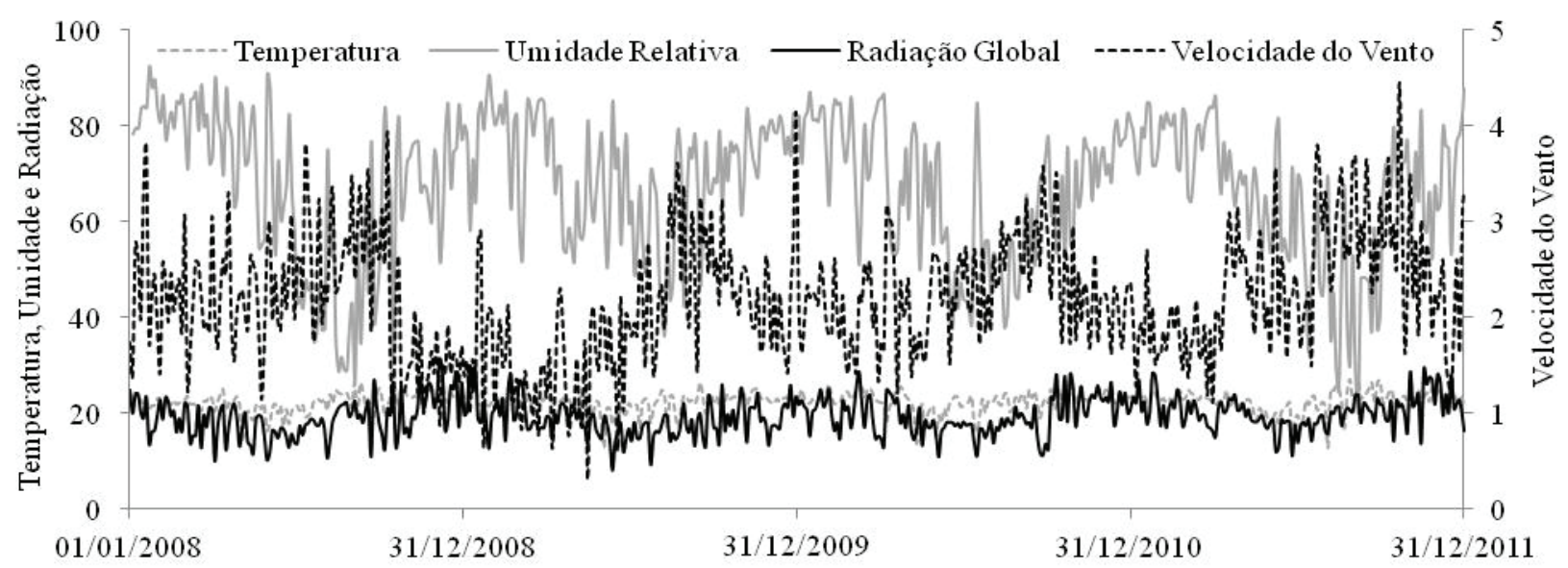

Figura 1. Variação diária da temperatura média $\left({ }^{\circ} \mathrm{C}\right)$, umidade relativa $(\%)$, radiação global $\left(\mathrm{MJ} \mathrm{m}^{-2} \mathrm{dia}^{-1}\right)$ e da velocidade do vento $\left(\mathrm{m} \mathrm{s}^{-1}\right)$ no período de janeiro de 2008 a dezembro de 2011 em Chapadão do Sul, MS. 

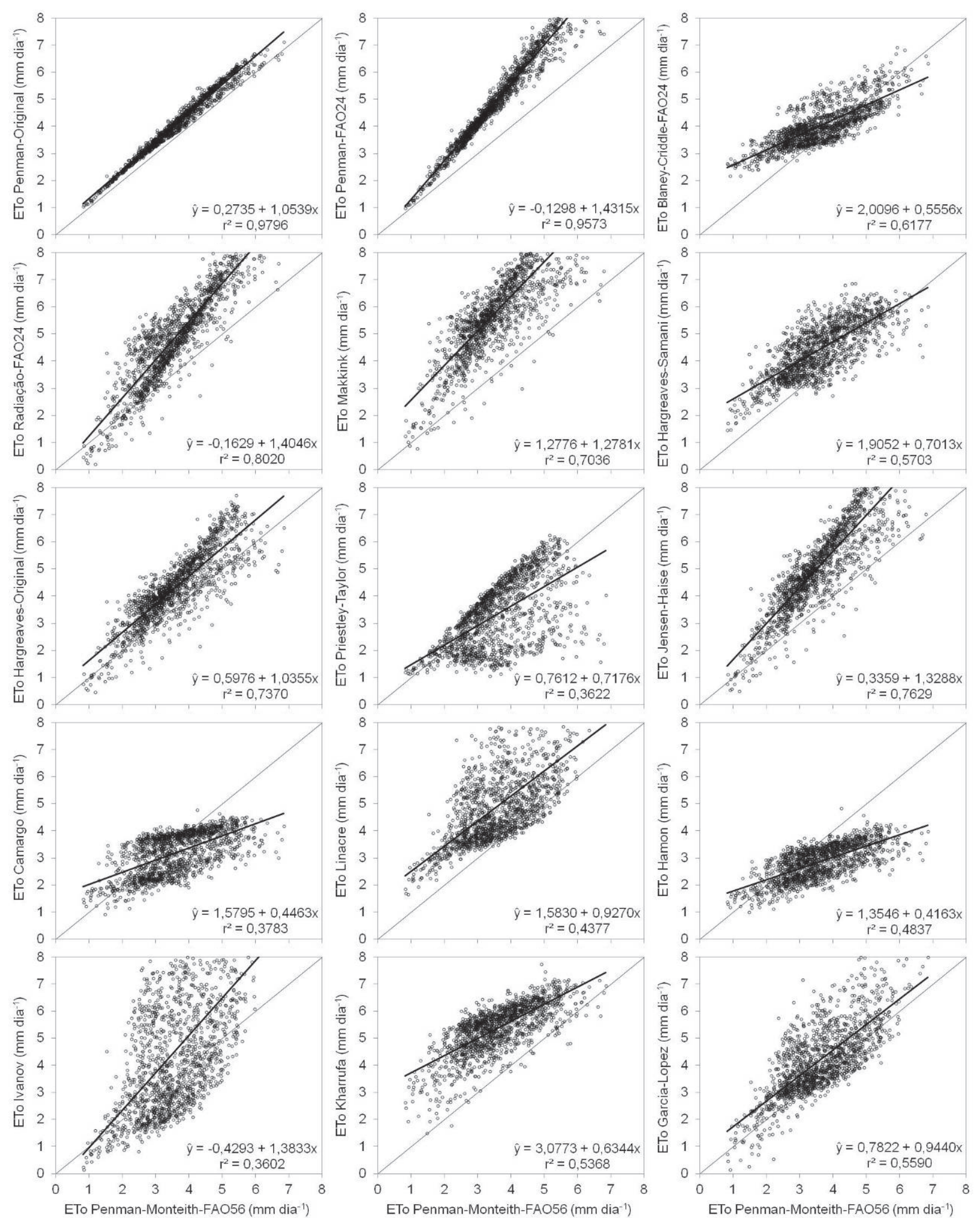

Figura 2. Valores de evapotranspiração de referência (ETo) obtidos pelo método de Penman-MonteithFAO56 comparados com os valores de ETo obtidos pelos métodos estudados.

\section{REVENG}

159-172p. ENGENHARIA NA AGRICULTURA, VIÇOSA - MG, V.21 N.2, MARÇO / ABRIL 2013 

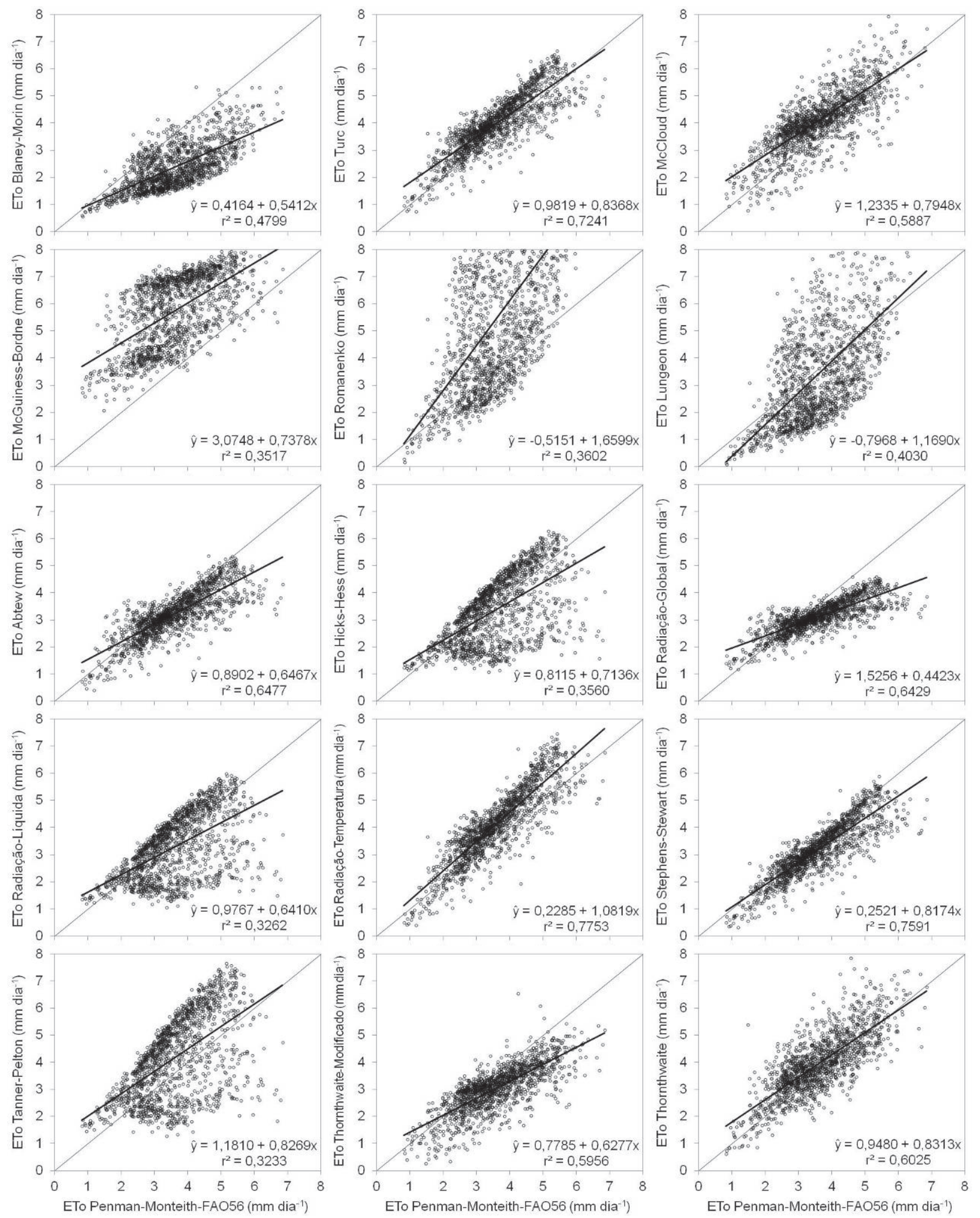

Figura 3. Valores de evapotranspiração de referência (ETo) obtidos pelo método de Penman-MonteithFAO56 comparados com os valores de ETo obtidos pelos métodos estudados. 
Observa-se também nas Figuras 2 e 3 que os métodos que apresentaram o melhor ajuste, de acordo com o coeficiente de determinação $\left(\mathrm{r}^{2}\right)$, foram os métodos Penman-Original $\left(\mathrm{r}^{2}=0,9796\right)$ e Penman-FAO24 $\left(r^{2}=0,9573\right)$, que utilizam os mesmos parâmetros de entrada que o método padrão. Entretanto, segundo Barros et al. (2009), a simples adoção do $\mathrm{r}^{2}$ como o único critério de definição da qualidade de métodos não é adequado, uma vez que este método não estabelece o tipo e a magnitude das diferenças entre um valor padrão e um valor previsto por modelos de estimativa. Os métodos Penman-Original e Penman-FAO24 também foram os que apresentaram o coeficiente de regressão "a" mais próximo de zero e coeficiente "b" mais próximo da unidade, confirmando sua superioridade entre os demais métodos para estimativa da ETo nas condições climáticas de Chapadão do Sul, MS. Os piores ajustes das equações para determinação da ETo, de acordo com o $\mathrm{r}^{2}$, foram para os métodos Priestley-Taylor $\left(r^{2}=0,3622\right)$, Camargo $\left(r^{2}=0,3783\right)$, Ivanov $\left(r^{2}\right.$ $=0,3602)$, McGuiness-Bordne $\left(\mathrm{r}^{2}=0,3517\right)$, Romanenko $\left(\mathrm{r}^{2}=0,3602\right)$, Hicks-Hess $\left(\mathrm{r}^{2}=0,3560\right)$, Radiação-Líquida $\left(\mathrm{r}^{2}=0,3262\right)$ e Tanner-Pelton $\left(\mathrm{r}^{2}\right.$ $=0,3233$ ). Dentre esses, muitos se caracterizam pela simplicidade de suas equações e pelo número reduzido de parâmetros de entrada. Apesar dessas vantagens, esses métodos citados não devem ser utilizados para estimativa da ETo para o Município de Chapadão do Sul, MS. Algumas equações, quando apresentam simplicidade em seus cálculos e parâmetros de entrada de fácil aquisição, recebem calibrações para serem utilizadas em determinada região. Entretanto, no caso das últimas equações citadas, essas não poderiam receber tal calibração devido a alta dispersão de seus valores em relação às retas 1:1 apresentadas nas Figuras 2 e 3 .

No Quadro 2 estão apresentados as estimativas do erro padrão (EEP), concordâncias de Willmott (d), correlações de Pearson (r), coeficientes de confiança (c) e desempenhos de Camargo e Sentelhas, obtidos das correlações entre os valores de evapotranspiração de referência (ETo) pelo método de Penman-Monteith-FAO56 com os obtidos pelos métodos estudados. Observa-se que o melhor modelo para estimativa da ETo para Chapadão do Sul, MS, de acordo com o EPE e o desempenho de Camargo e Sentelhas, foi o método de Penman-Original corroborando com Oliveira et al. (2001) em estudo realizado no Estado de Goiás, Pereira et al. (2009) em Minas Gerais, Bragança et al. (2010) no Espírito Santo e Cavalcante Jr. et al. (2011) no semi-árido nordestino. Apesar do método Penman-FAO24 ter apresentado $r^{2}$ satisfatório, seu valor de concordância de Willmott não obteve o mesmo sucesso, fazendo com que o seu desempenho (CAMARGO; SENTELHAS, 1997) fosse classificado apenas como "bom". Esse resultado pode ser explicado pelo fato dos valores estimados por Penman-FAO24 terem superestimado sensivelmente a ETo em relação ao método padrão em momentos de alta taxa evapotranspirométrica (Figura 2). Com isso, na comparação entre esses valores pontuais de ETo, houve uma redução no valor do índice de concordância. Alguns métodos receberam desempenho classificado como "muito bom", por ter conciliado altos valores de concordância de Willmott e correlação de Pearson, como Turc, Radiação-Temperatura e Stephens-Stewart. Além disso, esses métodos apresentaram baixos valores de EPE confirmando seu desempenho satisfatório. Entretanto, todos esses métodos precisam, além da temperatura, de dados de radiação global ou número de horas de brilho solar como parâmetros de entrada, dificultando a utilização em relação aos métodos que necessitam apenas de dados de radiação extraterrestre, temperatura e umidade relativa. É oportuno comentar que a radiação extraterrestre pode ser obtida apenas com a data e latitude do local, não necessitando, portanto, de dispositivos para sua medida. Alguns autores também verificaram estimativas satisfatórias de ETo utilizando os métodos de Turc, RadiaçãoTemperatura e Stephens-Stewart (REIS et al., 2007; BRAGANÇA et al., 2010; TAGLIAFERRE et al., 2010).

Os métodos Garcia-Lopes e McCloud receberam desempenho "bom", segundo Camargo e Sentelhas (1997). Algumas pesquisas corroboram com esse resultado (CAVALCANTE Jr. et al., 2011; KOSTINAKIS et al., 2011). Esses métodos são interessantes para serem utilizados em Chapadão do Sul, MS, uma vez que não necessitam de dados de radiação solar como parâmetros de entrada. O 
Quadro 2. Estimativa do erro padrão (EEP), concordância de Willmott (d), correlação de Pearson (r), coeficiente de confiança (c) e desempenho de Camargo e Sentelhas, obtidos das correlações entre os valores de evapotranspiração de referência (ETo) estimada pelos métodos estudados, com os valores estimados pelo método de Penman-Monteith-FAO56, em Chapadão do Sul, MS

\begin{tabular}{|c|c|c|c|c|c|c|}
\hline Método & ETo & EPE & $\mathrm{d}$ & $\mathrm{r}$ & $\mathrm{c}$ & Desempenho \\
\hline Penman-Monteith-FAO56 & 3,6024 & & & & & \\
\hline Penman-Original & 4,0699 & 0,4971 & 0,9495 & 0,9903 & 0,9403 & ótimo \\
\hline Penman-FAO24 & 5,0271 & 1,5278 & 0,7394 & 0,9792 & 0,7240 & bom \\
\hline Blaney-Criddle-FAO24 & 4,0112 & 0,7700 & 0,8152 & 0,8508 & 0,6936 & bom \\
\hline Radiação-FAO24 & 4,8970 & 1,5456 & 0,7380 & 0,9152 & 0,6754 & bom \\
\hline Makkink & 5,8819 & 2,4566 & 0,5433 & 0,8697 & 0,4725 & mau \\
\hline Hargreaves-Samani & 4,4316 & 1,0915 & 0,7483 & 0,8388 & 0,6277 & mediano \\
\hline Hargreaves-Original & 4,3279 & 0,9732 & 0,8341 & 0,8853 & 0,7384 & bom \\
\hline Priestley-Taylor & 3,3463 & 1,0709 & 0,7641 & 0,7860 & 0,6005 & sofrível \\
\hline Jensen-Haise & 5,1228 & 1,7411 & 0,6814 & 0,8971 & 0,6113 & mediano \\
\hline Camargo & 3,1873 & 0,9311 & 0,7231 & 0,7863 & 0,5686 & sofrível \\
\hline Linacre & 4,9225 & 1,7199 & 0,6267 & 0,7879 & 0,4938 & mau \\
\hline Hamon & 2,8543 & 1,0656 & 0,6739 & 0,8092 & 0,5453 & sofrível \\
\hline Ivanov & 4,5537 & 2,1888 & 0,5959 & 0,7754 & 0,4620 & mau \\
\hline Kharrufa & 5,3625 & 1,9039 & 0,5546 & 0,8144 & 0,4516 & mau \\
\hline Garcia-Lopez & 4,1828 & 1,0538 & 0,8019 & 0,8330 & 0,6680 & bom \\
\hline Blaney-Morin & 2,3662 & 1,4514 & 0,6204 & 0,8163 & 0,5065 & mau \\
\hline Turc & 3,9965 & 0,6906 & 0,8879 & 0,8795 & 0,7809 & muito bom \\
\hline McCloud & 4,0966 & 0,8798 & 0,8289 & 0,8331 & 0,6905 & bom \\
\hline McGuiness-Bordne & 5,7327 & 2,3902 & 0,4809 & 0,7806 & 0,3754 & péssimo \\
\hline Romanenko & 5,4645 & 3,0509 & 0,4862 & 0,7754 & 0,3770 & péssimo \\
\hline Lungeon & 3,4143 & 1,5116 & 0,7154 & 0,7822 & 0,5596 & sofrível \\
\hline Abtew & 3,2198 & 0,7297 & 0,8486 & 0,8492 & 0,7207 & bom \\
\hline Hicks-Hess & 3,3823 & 1,0713 & 0,7628 & 0,7843 & 0,5983 & sofrível \\
\hline Radiação-Global & 3,1190 & 0,8328 & 0,7582 & 0,8479 & 0,6429 & mediano \\
\hline Radiação-Líquida & 3,2857 & 1,0823 & 0,7455 & 0,7756 & 0,5782 & sofrível \\
\hline Radiação-Temperatura & 4,1258 & 0,8081 & 0,8818 & 0,9023 & 0,7956 & muito bom \\
\hline Stephens-Stewart & 3,1966 & 0,6585 & 0,8951 & 0,8954 & 0,8015 & muito bom \\
\hline Tanner-Pelton & 4,1598 & 1,3825 & 0,6895 & 0,7747 & 0,5342 & sofrível \\
\hline Thornthwaite-Modificado & 3,0395 & 0,8728 & 0,7981 & 0,8364 & 0,6676 & bom \\
\hline Thornthwaite & 3,9426 & 0,8041 & 0,8564 & 0,8422 & 0,7212 & bom \\
\hline
\end{tabular}

método de Garcia-Lopes precisa apenas de dados de temperatura média e umidade relativa, enquanto que o método McCloud, apenas da temperatura média do ar. Em relação a equação de GarciaLopes, a equação McCloud deve ser preferida para estimativa da ETo em Chapadão do Sul, MS. Além de sua aplicação ser mais simples, por necessitar apenas de um parâmetro de entrada, a equação de McCloud apresentou melhor ajuste de equação (Figuras 3), menor EPE e maior coeficiente de confiança em relação ao método de Garcia-Lopez. Essa equação foi desenvolvida por McCloud em 1955, para obtenção da evapotranspiração potencial de hipódromos e de campos de golfe, sendo ainda utilizada em muitos países.

Os métodos de Blaney-Criddle-FAO24, Radiação-FAO24, Hargreaves-Original, Abtew, Thornthwaite-Modificado e Thornthwaite receberam desempenho "bom", segundo Camargo e Sentelhas (1997). O método de Blaney-Criddle-FAO24 é 
bastante utilizado pelos agricultores no semi-árido e pode ser utilizado em Chapadão do Sul, MS. Entretanto, necessita de algumas informações, que normalmente são extraídas de tabelas, como porcentagem de horas de sol. Quando essas informações são utilizadas incorretamente, podem levar a erros de grande magnitude na estimativa de ETo. O método de Radiação-FAO24 foi derivada do método de Makkink, e como observado na presente pesquisa, também apresenta boas estimativas de ETo em várias regiões do País, como observado por Oliveira et al. (2008), Pereira et al. (2009), Mendonça e Dantas (2010), Tagliaferre et al. (2010), Alencar et al. (2011) e Cavalcante Jr. et al. (2011). Entretanto, esse método juntamente com Hargreaves-Original e Abtew, depende da radiação global, tornando seu uso dificultado como relatado anteriormente para os métodos de Turc, RadiaçãoTemperatura e Stephens-Stewart. Os métodos de Thornthwaite-Modificado e Thornthwaite podem ser utilizados na região em estudo. Entretanto, as constantes "a" e "I", necessárias para a estimativa da ETo, foram retiradas de apenas quatro anos de série histórica, pois a estação meteorológica de Chapadão do Sul ainda não apresenta normal climatológica de 30 anos, o que seria recomendável. Dentre todos os métodos estudados na presente pesquisa, as equações Thornthwaite-Modificado e Thornthwaite são as únicas equações físicas. Outros pesquisadores também encontraram bom desempenho desses métodos, como apresentado por Silva et al. (2005), Back et al. (2008) e Syperreck et al. (2008).

O método de Hargreaves-Samani é muito utilizado para estimativa da ETo no País, e apesar de ter apresentado baixo valor de EPE na presente pesquisa, recebeu desempenho classificado como "mediano", não sendo, dessa forma, recomendado para estimativas de ETo em Chapadão do Sul, corroborando com Oliveira et al. (2008) para Viçosa-MG, Reis et al. (2007) para Venda Nova do Imigrante-ES, Back et al. (2008) em UrussangaSC, Bragança et al. (2010) em Cachoeiro do Itapemerim, Sooretama e Venda Nova-ES, Tagliaferre et al. (2010) em Eunápolis-BA e Alencar et al. (2011) em Uberaba-MG. Entretanto, por ser bastante simples, necessitando apenas de dados de temperatura máxima e mínima, espera- se que a mesma metodologia seja objeto de estudo no futuro próximo, já que apresentou razoável coeficiente de determinação (Figura 2). Dessa forma, poder-se-á obter calibração do método para que aqueles produtores sem condições de adquirirem estações meteorológicas completas, possam obter estimativas confiáveis de ETo para o manejo correto da irrigação, utilizando apenas um termômetro de máxima e mínima.

Os métodos Makkink, Priestley-Taylor, Camargo, Linacre, Hamon, Ivanov, Kharrufa, Blaney-Morin, McGuiness-Bordne, Romanenko, Lungeon, Hicks-Hess, Radiação-Líquida e TannerPelton receberam desempenho "sofrível", "mau" ou "péssimo" e não devem ser utilizados no Município de Chapadão do Sul, MS. O método Makkink foi desenvolvido para as condições climáticas de Wageningem, na Holanda, e provavelmente esse foi o fator para tal desempenho. Outros pesquisadores também verificaram o mesmo comportamento em suas pesquisas (ARAÚJO et al., 2007; BARROS et al., 2009; CAVALCANTE Jr. et al., 2011). O método Priestley-Taylor foi desenvolvido para estimativa da evaporação de superfícies saturadas em uma atmosfera não saturada, que é a condição normal da natureza, entretanto seus coeficientes não se ajustaram as condições climáticas da região de estudo corroborando com Barros et al. (2009) e Cavalcante Jr. et al. (2011). O método de Camargo é derivado do método de Thornthwaite, e funciona eficazmente para regiões de clima tropical e equatorial úmidos. Entretanto, para Chapadão do Sul não obteve-se boas estimativas de ETo, corroborando com Cavalcante Jr. et al. (2011). O método Linacre é derivado do método de Penman, estimando a evapotranspiração por base de dados geográficos (latitude e altitude) e temperatura, entretanto, possivelmente a desconsideração de radiação e velocidade do vento proporcionou esse desempenho não satisfatório corroborando com Pereira et al. (2009) e Mendonça e Dantas (2010). Muitos trabalhos corroboram com a presente pesquisa em relação ao desempenho não satisfatório do método de Hamon (BORGES; MEDIONDO, 2007; PEREIRA et al., 2009; CAVALCANTE Jr. et al., 2011). O método de Hamon apesar da complexidade de apresentar muitos coeficientes necessita apenas de um 
parâmetro medido (temperatura média do ar). O método de Ivanov é simples e foi desenvolvido para estimativa de ETo para períodos mínimos de um mês. Dorfman (1977), adaptou a equação para cálculo de ETo diária e como verificado, não apresentou desempenho satisfatório para Chapadão do Sul, corroborando com a pesquisa de Back et al. (2008). O método de Kharrufa utiliza os mesmos parâmetros que o método de Blaney-Criddle-FAO24, porém não apresentou o mesmo desempenho, principalmente devido a baixa concordância de Willmott, corroborando com os resultados de Borges e Mediondo (2007) e Pereira et al. (2009). Os métodos Lungeon e Tanner-Pelton, como o método Makkink, também utiliza coeficientes obtidos em outros países, e possivelmente explica o desempenho classificado como "sofrível". O método Tanner-Pelton é baseado no saldo de radiação, e suas constantes foram obtidas para as condições do estado de Wisconsin, Estados Unidos.

\section{CONCLUSÕES}

Diante dos resultados, concluiu-se que:

- Em ordem, os melhores métodos para estimativa da evapotranspiração de referência para Chapadão do Sul, MS, são: PenmanOriginal, Stephens-Stewart, RadiaçãoTemperatura, Turc, Hargreaves-Original, Penman-FAO24, Thornthwaite, Abtew, Blaney-Criddle-FAO24, McCloud, RadiaçãoFAO24, Garcia-Lopez, ThornthwaiteModificado, Radiação-Global, HargreavesSamani e Jensen-Haise;

- Quando se tem apenas dados de temperatura, recomenda-se utilizar o método de McCloud para estimativa da evapotranspiração de referência em Chapadão do Sul;

- Os métodos Makkink, Priestley-Taylor, Camargo, Linacre, Hamon, Ivanov, Kharrufa, Blaney-Morin, McGuiness-Bordne, Romanenko, Lungeon, Hicks-Hess, RadiaçãoLíquida e Tanner-Pelton não devem ser utilizados para a estimativa da evapotranspiração de referência em Chapadão do Sul.

\section{REFERÊNCIAS BIBLIOGRÁFICAS}

ALENCAR, L.P.; DELGADO, R.C.; ALMEIDA, T.S.; WANDERLEY, H.S. Comparação de diferentes métodos de estimativa diária da evapotranspiração de referência para a região de Uberaba. Revista Brasileira de Ciências Agrárias, Recife, v.6, n.2, p.337-343, 2011.

ALLEN, R.G.; JENSEN, M.E.; WRIGHT, J.; BURMAN, R.D. Operational estimates of reference evapotranspiration. Agronomy Journal, Madison, v.81, n.4, p.650-662, 1989.

ALLEN，R.G.; PEREIRA， L.S.; RAES，D.; SMITH, M. Crop evapotranspiration: guidelines for computing crop water requirements. Rome: FAO, 1998. 300p. (Irrigation and Drainage Paper, 56).

ARAÚJO, W.F.; COSTA, S.A.A.; SANTOS, A.E. Comparação entre métodos de estimativa da evapotranspiração de referência (ETo) para Boa Vista-RR. Caatinga, Mossoró, v.20, n.4, p. 84-88, 2007.

BACK, A.J. Desempenho de métodos empíricos baseados na temperatura do ar para a estimativa da evapotranspiração de referência em Urussanga, SC. Irriga, Botucatu, v.13, n.4, p.449-466, 2008.

BARROS, V.R.; SOUZA, A.P.; FONSECA, D.C.; SILVA, L.B.D. Avaliação da evapotranspiração de referência na região de Seropédica, Rio de Janeiro, utilizando lisímetro de pesagem e modelos matemáticos. Revista Brasileira de Ciências Agrárias, Recife, v.4, n.2, p.198-203, 2009.

BERLATO, M. A.; MOLION, L. C. B. Evaporação e evapotranspiração. Porto Alegre: IPAGRO/ Secretaria de Agricultura, 1981. 95 p. (Boletim Técnico, 7).

BLANEY, H.F.; MORIN, K.V. Evaporation and consumptive use of water: empirical formulas. Transaction American Geophysical Union, Washington, v.23, n.1, p.76-83, 1942. 
BORGES,A.C.; MENDIONDO, E.M. Comparação entre equações empíricas para estimativa da evapotranspiração de referência na Bacia do Rio Jacupiranga. Revista Brasileira de Engenharia Agrícola e Ambiental, Campina Grande, v.11, n.3, p.293-300, 2007.

BRAGANÇA, R.; REIS, E.F.; GARCIA, G.O.; PEZZOPANE, J.E.M. Estudo comparativo da estimativa da evapotranspiração de referência no período chuvoso para três localidades no estado do Espírito Santo. Idesia, Arica, v.28, n.2, p.21-29, 2010.

BRUIN, H.A.R.; KEIJMAN, J.Q. The PriestleyTaylor evaporation model applied to large, shallow lake in the Netherlands. Journal of Applied Meteorology, New York, v.18, n.7, p.898-903, 1979.

BRUIN, H.A.R.; STRICKER, J.N.M. Evaporation of grass under non-restricted soil moisture conditions. Hydrological Sciences Journal, London, v.45, n.3, p.391-406, 2000.

CAMARGO, A.P. Balanço hídrico no estado de São Paulo. 3. ed. Campinas: IAC, 1971. 24p. (Boletim, n. 116).

CAMARGO, A.P.; SENTELHAS, P.C. Avaliação do desempenho de diferentes métodos de estimativa da evapotranspiração potencial no estado de São Paulo. Revista Brasileira de Agrometeorologia, Santa Maria, v.5, n.1, p.89-97, 1997.

CAMARGO, A.P.; MARIN, F.R.; SENTELHAS, P.C.; PICINI, A.G. Ajuste da equação de Thornthwaite para estimar a evapotranspiração potencial em climas áridos e super-úmidos, com base na amplitude térmica diária. Revista Brasileira de Agrometeorologia, Santa Maria, v. 7, n. 2, p. 251-257, 1999.

CAVALCANTE Jr., E.G.; OLIVEIRA, A.D.; ALMEIDA, B.M.; ESPÍNOLA SOBRINHO, J. Métodos de estimativa da evapotranspiração de referência para as condições do semiárido Nordestino. Semina, Londrina, v.32, n. suplemento, p.1699-1708, 2011.
CHAPADÃO DO SUL. Base Econômica. < http:// www.chapadaodosul.ms.gov.br $>$ Acesso em: 4 mar. 2013.

COHEN, J. Statistical power analysis for the behavioral sciences. New Jersey: Lawrence Erlbaum, 1988. 569p.

DOORENBOS, J.; PRUITT, W.O. Crop water requirements. Rome: FAO, 1977. 143p. (Irrigation and Drainage paper, 24).

DORFMAN, R. Critérios de avaliação de alguns métodos de cálculo da evapotranspiração potencial. Porto Alegre. UFRGS. Dissertação de Mestrado, 1977. 210p.

EJIEJI, C.J. Performance of three empirical reference evapotranspiration models under three sky conditions using two solar radiation estimation methods at Ilorin, Nigeria. Agricultural Engineering International, Beijing, v.13, n.3, p.1-21, 2011.

GARCIA, B.J.; LOPEZ, D. Formula para el caçulo de la evapotranspiracion potencial adaptada al tropico (15 $\mathrm{N}$ - $\left.15^{\circ} \mathrm{S}\right)$. Agronomia Tropical, Maracay, v.20, n.5, p.335-345, 1970.

HAMON, W.R. Estimating potential evapotranspiration. Journal of Hydraulics Division ASCE, New York, v.87, n.3, p.107-120, 1961.

HARGREAVES, G.H. Estimation of potential and crop evapotranspiration. Transactions of the ASAE, Saint Joseph, v.17, n.1, p.701-704, 1974.

HARGREAVES, G.L.; SAMANI, Z.A. Reference crop evapotranspiration from temperature. Basin. Journal of the Irrigation and Drainage Division ASCE, New York, v.111, n.1, p.113-124. 1985.

JACOBS, J.M.; SATTI, S.R. Evaluation of reference evapotranspiration methodologies and AFSIRS crop water use simulation model (Final Report). Gainesville: Department of Civil and Coastal Engineering, University of Florida, 2001. 114p. 
JENSEN, M.E.; HAISE, H.R. Estimating evapotranspiration from solar radiation. Journal of the Irrigation and Drainage Division ASCE, New York, v.4, n.1, p.15-41, 1963.

KHARRUFA, N.S. Simplified equation for evapotranspiration in arid regions. Beiträge zur Hydrologie, Kirchzarten, v.5, n.1, p.39-47, 1985.

KOSTINAKIS, K.; XYSTRAKIS, F.; THEODOROPOULOS, K.; STATHIS, D.; ELEFTHERIADOU, E.; MATZARAKIS, A. Estimation of reference potential evapotranspiration with focus on vegetation science - the EmPEst software. Journal of Irrigation and Drainage Engineering, New York, v.137, n.9, p.616-619, 2011 .

LINACRE, E.T.A simple formula for estimating evapotranspiration rates in various climates, using temperature data alone. Agricultural Meteorology, Amsterdam, v.18, n.1, p.409-424, 1977.

MAKKINK, G.F. Testing the Penman formula by means of lysimeters. Journal of the Institution of Water Engineers, New York, v.11, n.3, p.277-288. 1957.

MENDONÇA, E.A.; DANTAS, R.T. Estimativa da evapotranspiração de referência no município de Capim, PB. Revista Brasileira de Engenharia Agrícola e Ambiental, Campina Grande, v.14, n.2, p.196-202, 2010.

OLIVEIRA, L.F.C.; CARVALHO, D.F.; ROMÃO, P.A.; CORTÊS, F.C. Estudo comparativo de modelos de estimativa da evapotranspiração de referência para algumas localidades no estado de Goiás e Distrito Federal. Pesquisa Agropecuária Tropical, Goiânia, v.31, n.2, p.121-126, 2001.

OLIVEIRA, R.A.; TAGLIAFERRE, C.; SEDIYAMA, G.C.; MATERAM, F.J.V.; CECON, P.R. Desempenho do Irrigâmetro na estimativa da evapotranspiração de referência. Revista Brasileira de Engenharia Agrícola e Ambiental, Campina Grande, v.12, n.2, p.166-173, 2008.
OUDIN, L.; MICHEL, C.; ANCTIL, F. Which potential evapotranspiration input for a lumped rainfall-runoff model?: Part 1-Can rainfallrunoff models effectively handle detailed potential evapotranspiration inputs? Journal of Hydrology, Amsterdam, v.303, n.1-4, p.290-306, 2005.

PEÑA, L.S.; PEÃN, E.P. Obtención de modelos para la determinación de la evapotranspiración de referencia en condiciones de clima subhúmedo seco en las tunas, cuba. Idesia, Arica, v.28, n.1, p.55-59, 2010

PENMAN, H.L. Natural evaporation from open water, bare soil and grass. Proceedings of Royal Society-Series A, London, v.193, n.1, p.120-145, 1948.

PEREIRA, D.R.; YANAGI, S.N.M.; MELLO, C.R.; SILVA, A.M.; SILVA, L.A. Desempenho de métodos de estimativa da evapotranspiração de referência para a região da Serra da Mantiqueira, MG. Ciência Rural, Santa Maria, v.39, n.9, p.2488-2493, 2009.

PRIESTLEY, C.H.B.; TAYLOR, R.J. On the assessment of surface heat flux and evaporation using large-scale parameters. Monthly Weather Review, Boston, v.100, n.2, p.81-92, 1972.

REIS, E.F.; BRAGANÇA, R.; GARCIA, G.O. Estudo comparativo da estimativa da evapotranspiração de referência para três localidades do Estado do Espírito Santo no período seco. Idesia, Arica, v.25, n.3, p.75-7854, 2007.

SILVA, V.P.R.; BELO FILHO, A.F.; SILVA, B.B.; CAMPOS, J.H.B.C. Desenvolvimento de um sistema de estimativa da evapotranspiração de referência. Revista Brasileira de Engenharia Agrícola e Ambiental, Campina Grande, v.9, n.4, p.547-553, 2005.

SOBEL, T.F.; MUNIZ, A.L.P.; COSTA, E.F. Divisão regional do desenvolvimento humano no mato grosso do sul: uma aplicação da análise de cluster. Análise, Porto Alegre, v.20, n.1, p.67-83, 2009. 
SYPERRECK, V.L.G.; KLOSOWSKI, E.S.; GRECO, M.; FURLANETTO, C. Avaliação de desempenho de métodos para estimativas de evapotranspiração de referência para a região de Palotina, Estado do Paraná. Acta Scientiarum Agronomy, Maringá, v.30, n.5, p.603-609, 2008.

TAGLIAFERRE, C.; SILVA, R.A.J.; ROCHA, F.A.; SANTOS, L.C.; SILVA, C.S. Estudo comparativo de diferentes metodologias para determinação da evapotranspiração de referência em Eunápolis-BA. Caatinga, Mossoró, v.23, n.1, p.103-111, 2010.

THORNTHWAITE, C.W. An approach toward rational classification of climate. The Geographical Review, New York, v.38, n.1, p.55-94, 1948.

TOMAR, V.S.; O'TOOLE, J.C. Water use in lowland rice cultivation in Asia: a review of evapotranspiration. Agricultural Water Management, New York, v.3, n.1, p.83-106, 1980.
TURC, L. Evaluation des besoins en eau d'irrigation, évapotranspiration potentielle, formule simplifiée et mise à jour. Annals of Agronomy, v.12, n.1, p.13-49, 1961.

WILLMOT, C.J.; ACKLESON, S.G.; DAVIS, J.J.; FEDDEMA, K.; KLINK, D.R. Statistics for the evaluation and comparison of models. Journal of Geophysical Research, Ottawa, v.90, n.5, p.89959005, 1985.

WINTER, T.C.; ROSENBERRY, D.O; STURROCK, A.M. Evaluation of 11 equations for determining evapotranspiration for a small lake in the north central United States. Water Resources Research, v.31, n.4, p.983-993, 1995.

ZIMMERMANN, E.D. Evaporación, transpiración y evapotranspiración en Orsolini. In: ZIMMERMANN, E. D.; BASILE, P. A. (eds.). Hidrologia: Procesos y Métodos. Rosario: UNR Editora, 2000, p.65-89. 